\title{
Huellas italianas de Emilia Pardo Bazán: epistolario, prensa y viaje
}

\author{
Emilia Di Bono \\ emiliadibono@gmail.com
}

(recibido setembro/2013, revisado setembro/2013)

RESUMEN: Epistolario, prensa y viaje: tres palabras que permiten comprender la vida y la obra de la escritora coruñesa Emilia Pardo Bazán. Vittorio Pica, Anna Radius Zuccari y Luigi Capuana son solo algúnos de los intelectuales italianos que mantuvieron relaciones epistolares con la condesa: reconstruir las amistades culturales de la autora es el punto de partida para las investigaciones futuras sobre este tema.

Estudiando los textos presentes en la Real Academia Galega, el tema del viaje gran atención ha adquirido, que permite a la condesa comparar la situación de su país con los otros, intentando comunicar al lector sus pensamientos. Una de sus primeras exploraciones fue por las ciudades españolas, francesas y en el norte de Italia, y sus anotaciones se encuentran en los Apuntes de un viaje. De España a Ginebra, redactados en 1873. Más tarde su pasión por el viaje la llevó a entrar en contacto con las principales revistas españolas, considerando la prensa come el medio más eficaz de difusión.

Con respecto a la amplia producción de la obra pardobazaniana los datos encontrados hasta ahora son muy pocos, así como en las traducciones al italiano de cuentos, novelas y ensayos, trabajo Ilevado a cabo por los profesosres Danilo Manera (Universidad de Milán), Laura Silvestri (Universidad de Roma y Udine) y Tonina Paba (Universidad de Cagliari). El objectivo de esta investigación es la creación de un plan de colaboración para la difusión del inmenso tesoro literario de la condesa Doña Emilia.

PALABRAS CLAVE: Epistolario, prensa, viaje, investigación, colaboración

ABSTRACT: Epistolary, printing, travelling, these are three keywords which allow us to fully understand and appreciate the life and the work of Emilia Pardo Bazan. Vittorio Pica, Anna Radius Zuccari e Luigi Capuana are just some of the brilliant minds that had shared letters and writings with the countess: in order to begin a study about her work, it is necessary to retrace her cultural and social relationships. By studying the texts kept at the "Emilia Pardo Bazan Museum" and at the "Real Academia Galega" we can understand the importance of the topic of travelling thanks which she made brilliant comparisons between countries and tried to explain her thoughts and ideas about it to the readers.

One of her first "expedition" was in the Spanish, French and Nothern Italian cities and her notes are gathered in Apuntes de un viaje. De España a Ginebra publishes in 1873. Later she began to get interested to the principal Spanish periodicals and she began to evaluate the publishing industry as one of the most strong media. 
Despite the enormous amount of her production, the information collected are not many, as much as Italian translation of the short stories, novels and essays. Danilo Manera (University of Milan), Laura Silvestri (University of Rome and Udine) and Tonina Paba (University of Cagliari) are the major scholars of the production of the countess-writer. The main purpose of this research is the creation of a collaboration plan for the diffusion of the enormous literary work of the countess Donna Emilia

KEY WORDS: Epistolary, printing, travelling, collaboration, research.

Reconstruir las relaciones de Emilia Pardo Bazán con Italia entre finales del siglo XIX y primeras décadas del XX es algo difícil, aunque sean abundantes y significativas. Algunos trabajos, llevados a cabo por investigadores italianos, han adquirido gran importancia, ya que analizan las amistades de doña Emilia con intelectuales italianos de la época, corroboradas por las numerosas cartas encontradas en su epistolario ${ }^{1}$, y trazan las líneas de las publicaciones de las obras de Emilia Pardo Bazán en lengua italiana durante el siglo $X X^{2}$. Quien se ocupe de la producción literaria de doña Emilia no puede no centrarse en la pasión de la escritora por la narración de viajes, siempre viva y constante, Ilevándola a peregrinar por diferentes ciudades italianas, aclarada por el hecho de que fue enviada al extranjero por cuenta de El Imparcial, del que procede su obra Mi romería (1888), que se estructura como una especie de diario de viaje en tren de Madrid a Roma.

Por esta razón, mi trabajo sigue tres directrices: el análisis de las amistades de doña Emilia con los escritores e intelectuales italianos de la época, el reflejo de su obra en Italia y su actividad como periodista-viajera. La imposibilidad de investigar en persona en los archivos y en las bibliotecas italianas limita este estudio a algunos archivos disponibles en Internet y a la lectura de los textos analizados en la Real Academia Galega. Por lo tanto, se puede considerar mi trabajo como un estudio introductorio a este tema, quizás utilizado en el futuro como punto de partida para otras investigaciones.

Como ya queda dicho, la correspondencia de Emilia Pardo Bazán con algunos intelectuales italianos de la época nos permite demostrar cómo la escritora fue no sólo conocida en Italia, sino más bien admirada y considerada una de las máximas autoridades literarias de su época.

La primera referencia encontrada en la lectura de los textos es la presencia del intercambio de correspondencia con el escritor Salvatore Farina, narrador, muy

\footnotetext{
1 Paba, Tonina, “Emilia Pardo Bazán e Italia”, en La literatura de Emilia Pardo Bazán, José Manuel González Herrán, Cristina Patiño Eirín, Ermitas Penas Varela (eds.), Fundación Caixa Galicia, CasaMuseo Emilia Pardo Bazán, Real Academia Galega, 2009, pp. 527-536.

2 Manera, Danilo, “Emilia Pardo Bazán en lengua italiana”, en Emilia Pardo Bazán: estado de la cuestión, José Manuel González Herrán, Cristina Patiño Eirín, Ermitas Penas Varela (eds.), Real Academia Galega, 2005, pp. 363-365.
} 
famoso en su tiempo, del Reino de Cerdeña y del Reino de Italia. Siendo el director de la Rivista Minima ${ }^{3}$, pudo destacar la figura de la pionera escritora:

Salutiamo una scrittrice valorosa che si è manifestata da poco in Ispagna, dove non è, come da noi, abbondanza di scrittrici. Si chiama Emilia Pardo Bazán, e dopo aver chiamato l'attenzione sopra di sé con un bel racconto: Un viaje de novios, ha ora affermato il suo splendido ingegno in uno studio critico di molto valore, intitolato: La cuestión palpitante, in cui si parla di verismo, di naturalismo, di verità e di natura (che sono molto di più)... ${ }^{4}$.

De hecho, a partir de 1878, el interés que doña Emilia había venido demostrando por la novela realista se manifestó claramente en La cuestión palpitante, en el que la escritora habla de lo que debe ser la novela contemporánea, porque acepta la fórmula realista, ya que es la única forma válida para crear una novela moderna ${ }^{5}$. Los ecos de la polémica Ilegaron a Italia, y Salvatore Farina, siendo un hombre que contaba en el panorama de las letras italianas e interesado en los problemas teóricos de la literatura ${ }^{6}$, acogió con interés las teorías de la Pardo Bazán. Por esta razón, decidió pedirle uno de sus artículos para traducirlo en su revista: se trata de una carta de 1883 en que Farina pide a doña Emilia permiso para publicar en su revista el capítulo XIX de La cuestión palpitante; se supone que el capítulo XVIII obraba ya en su poder y no se conoce si el capítulo XIX le Ilegó posteriormente, ya que unicamente acabó publicándose en Italia el capítulo XVIII, titulado En España ${ }^{7}$. Lo importante es que la traducción del artículo de la escritora al italiano fue rápida, para que en Italia se conociera lo que doña Emilia tenía que decir sobre la literatura española de su época.

Salvatore Farina no fue el único en mantener relaciones de correspondencia con Emilia Pardo Bazán. El interés hacia la doctrina naturalista Ilamó también la atención de Vittorio Pica, joven intelectual napolitano, y uno de los fundadores de la revista quincenal Fantasio de Nápoles (1881-1883), que presenta a la escritora a los lectores italianos, utilizando palabras muy amables, definiéndola como

\footnotetext{
3 Revista italiana dirigida por Salvatore Farina de 1872 hasta 1883.

4 Navarro Salazar, Ma Teresa, "La cuestión palpitante en Italia: Salvatore Farina y Emilia Pardo Bazán”, en Epos (6) (Publicación de la UNED), Madrid, 1990, pp. 551-559, p. 555. "Saludamos a una escritora valiente, recientemente manifesta en España, en que no hay abundancia de escritoras, como en Italia. Se llama Emilia Pardo Bazán, y después de haber llamado la atención con el cuento [sic.] Un viaje de novios, ahora afirma su hermoso talento en un estudio crítico de alto nivel y valor, titulado La cuestión palpitante, en que se habla de verismo, de naturalismo, de verdad y de naturaleza (que son mucho más)".

5 Ibid., p. 553.

6 Teorías que había expuesto en un artículo aparecido en la Rivista Minima con el título "Alcune idee sul romanzo" en 1872 .

7 Navarro Salazar, Ma Teresa, ob. cit., p. 559.
} 
valiente novelista española. La escritora gallega muestra conocer el libro de Pica All'avanguardia (1890), del que habla en un artículo publicado en la revista La España Moderna, titulado Últimas modas literarias (sobre un libro italiano) en $1890^{8}$. En esta reseña subraya cómo la novela del Realismo español es el producto autóctono y la prolongación de la ilustre tradición nacional de un género encabezado por Don Quijote9. En la biblioteca de Doña Emilia no figura este libro, sino otro, Arte dell'Estremo Oriente de 1894, en la edición original. De la obra se guardan dos copias, las dos con dedicatoria autógrafa del autor: una en español ("A Doña Emilia Pardo Bazán afectuoso recuerdo de Vittorio Pica"), y otra en italiano "All'illustre scrittrice e gentile amica Doña Emilia Pardo Bazán con affetto Vittorio Pica"10).

En la misma obra, Pica dedica un capítulo a la novela en Rusia, e informa sobre los trabajos de doña Emilia al respecto, sobre todo considerando las conferencias que la escritora presidió en 1887 en el Ateneo de Madrid. El crítico afirma:

... esse debbono essere consultate da chiunque voglia formarsi un concetto completo ed esatto dell'odierna letteratura romanzesca russa ${ }^{11}$

añadiendo que la escritora estaba a punto de escribir un importante libro sobre Rusia. Todo esto supone que existía una correspondencia entre los dos, a pesar de que entre las cartas de Vittorio Pica hasta hoy no se hayan encontrado las de doña Emilia.

Gracias a la reconstrución de las relaciones entre Emilia Pardo Bazán e Italia, y sobre todo entre Emilia Pardo Bazán y Vittorio Pica, la doctora Tonina Paba ha encontrado otra referencia a la autora gallega: se trata de una carta del intelectual napolitano a la escritora Anna Radius Zuccari, conocida con el pseudonimo Neera ${ }^{12}$. No se han encontrado cartas enviadas a Neera por doña Emilia, pero sí que existe una carta del 14 de noviembre de 1886, en que Pica le pide a la escritora que envie sus libros (Marito dell'amica, Milano, 1885; Teresa, Milano,1886) a Doña Emilia, ya que estaba interesada en la producción literaria italiana.

\footnotetext{
${ }^{8}$ Paba, Tonina, “Emilia Pardo Bazán e Italia”, op. cit., p. 530.

9 Garosi, Linda, Poéticas de una crisis. La literatura italiana y española entre los siglos XIX y XX, Universidad de Córdoba, Facultad de Filosofía y Letras, Departamento de Traducción e Interpretación, Lenguas Romances, Estudios Semíticos y Documentación, 2007, p. 181.

10 Paba, Tonina, Emilia Pardo Bazán e Italia, ob. cit., p. 530. "A la ilustre escritora y amiga amable Doña Emilia Pardo Bazán con cariño Vittorio Pica”.

11 Ibid., p. 531. "Éstas tienen que ser consultadas por los que quieren tener un concepto completo y exacto sobre la literatura novelesca rusa actual".

12 Anna Radius Zuccari fue una escritora milanesa que con el pseudónimo horaciano de Neera publicó ensayos y novelas. Nacida en Milán en 1846, pasó momentos felices en Caravaggio, lugar donde vivían sus abuelos maternos, y que son recordados y descritos en sus obras de forma entrañable.
} 
Tonina Paba refiere las palabras de Pica:

La signora Emilia Pardo Bazán, valorosissima scrittrice spagnola, i cui romanzi ed i cui libri di critica hanno ottenuto il maggiore successo nella penisola iberica, desidera conoscere le più interessanti pubblicazioni dell'odierna letteratura italiana e perciò si è rivolta a me. Il Capuana ed il Verga le hanno già inviato i loro libri: vuole anche Ella inviare Teresa ed il Marito dell'amica? L'indirizzo della Pardo Bazán è La Coruña Galicia (Spagna) ${ }^{13}$.

De hecho, en la biblioteca de Doña Emilia, según el catálogo que consulta Tonina Paba para su trabajo (Fernández-Couto 2005 y Menéndez Rodríguez 2007) hay actualmente dos obras de Verga (Eros, 1884 y Storia di una capinera, 1893) y cuatro de Capuana (Ribbrezzo, 1885; Giacinta, 1886; Fosca, 1886 y Nuove paesane, 1898); las tres de Capuana (Ribbrezzo, Giacinta y Nuove paesane) Ilevan todas las dedicatorias a la escritora gallega:

Alla geniale scrittrice de Los Pazos de Ulloa in attestato di profonda ammirazione Luigi Capuana, Mineo 22 novembre $1886^{14 .}$

Alla valiente difensora della scuola naturalista Signora Emilia Pardo Bazán Omaggio di Luigi Capuana ${ }^{15}$.

A la illustra Signora Doña Emilia Pardo Bazán omaggio dell'autore, Roma, Via Curtatone, $6^{16}$.

No se acaban con estas las obras de escritores italianos presentes en la biblioteca de doña Emilia. Hay por ejemplo un libro de cuentos de Grazia Deledda, importante escritora y traductora, nacida en Cerdeña, y ganadora del Premio Nobel de Literatura en 1926.

La reconstrucción de las relaciones amistosas y culturales de la escritora es un trabajo muy importante, que se funda en el descubrimiento de indicios y elementos para llegar a delinear por completo el cuadro final. Indudablemente, la existencia de estas correspondencias es un punto de partida para las investigaciones futuras sobre este tema. Por cierto, se necesita un trabajo largo y minucioso para alcanzar

13 Paba, Tonina, op. cit., p. 532. “La señora Emilia Pardo Bazán, valiente escritora española, cuyas novelas y libros de crítica lograron mucho éxito en la Península Ibérica, quiere conocer las más interesantes publicaciones de la literatura italiana actual, y por eso se dirigió a mí. Capuana y Verga ya le enviaron sus libros: ¿quiere Usted enviarle Teresa y Marito dell'amica? La dirección de Pardo Bazán es La Coruña Galicia (España)".

14 Ibid., p. 533. "A la genial escritora de Los Pazos de Ulloa testimonio de profunda admiración Luigi Capuana, Mineo, 22 de noviembre de 1886 (Ribbrezzo)".

15 Ibid. "A la valiente defensora de la escuela naturalista Señora Emilia Pardo Bazán. Homanaje de Luigi Capuana (Giacinta)".

16 Ibid. "A la ilustre Señora Doña Emilia Pardo Bazán homenaje del autor, Roma, Via Curtatone, 6 (Nuove Paesane)". 
estos objetivos. Leyendo el estudio de Dolores Thion Soriano-Mollá, "El epistolario de Doña Emilia Pardo Bazán, estado de la cuestión"17, se conoce que hasta hoy existen en total ciento veintisiete cartas remitidas por Doña Emilia (el intervalo abarca de 1876 a 1920). Si bien la mayoría de estas cartas nunca se han encontrado hasta ahora, representan un punto fundamental para el estudio de la personalidad y de la aportación cultural de la condesa: de hecho, el epistolario es un testigo del mundo cultural, literario y artístico, que permite a los estudiosos penetrar en el universo interior de los escritores, facilitando la comprensión de sus pensamientos, de sus vidas y sobre todo de sus obras:

El epistolario nos la muestra en espíritu y en verdad. No escritas sus cartas ni con barruntos de que pudiesen ser publicadas nunca, son revelación franca de una psicología que no puede ser más poética... Y en tal sinceridad estriba en gran parte, el atractivo peculiar de su obra ${ }^{18}$.

Durante la lectura y el estudio de los numerosos textos presentes en la Real Academia, decidí llevar a cabo una pequeña investigación relacionada con el tema del viaje.

Según la definición de la Real Academia Española, el viaje es "un camino por donde se hace": esta experiencia, constante en Doña Emilia, le permite enfrentarse con gentes y culturas diferentes, comparar la situación de su propio país con los otros, y sobre todo intentar eliminar los tabues del bagaje cultural.

La habilidad de Doña Emilia reside en verificar las cosas en el sitio, hablar con personas que difieren en el color político y cultural, intentando comunicar al lector de su obra un único objetivo: el bien de la nación:

El viaje permite a la poliédrica personalidad de la condesa manifestarse en todas sus facetas, haciendo patente, además de una inagotable curiosidad, su brillante capacidad de observación y su aguda inteligencia, añadidas a una sólida preparación cultural y a la constante preocupación por no decepcionar las expectativas de sus lectores [...]. Lejos de mantenerse fiel a la tarea basada en la minuciosidad de las descripciones y en la objetividad de los datos ella opina que el viaje escrito es el alma del viajero, y por tanto, concede espacio a las propias reflexiones sobre lugares, hechos y personajes con los que tiene que ver ${ }^{19}$.

\footnotetext{
17 Thion Soriano-Mollá, Dolores, “El epistolario de Doña Emilia Pardo Bazán, estado de la cuestión”, en Emilia Pardo Bazán: estado de la cuestión, Simposio, 2-3 y 4 de xuño de 2004, José Manuel González Herrán, Cristina Patiño Eirín, Ermitas Penas Varela (eds.), Fundación Caixa Galicia, CasaMuseo Emilia Pardo Bazán, Real Academia Galega, 2005, pp. 181 - 217.

18 Ibid., p. 184. "La corrispondenza ce la mostra nello spirito e nella realtà. Le sue lettere non sono scritte con sentori di una loro mai pubblicazione... sono la franca rivelazione di una psicologia che non potrebbe essere più poetica... E su questa sincerità, in gran parte, si poggia l'attrazione peculiare della sua opera".

19 Pardo Bazán, Emilia, Viajes por Europa, introdución y cronología de T. Paba (Universidad de Cagliari), Editorial Bercimuel, Colmenar Viejo, Madrid, 2006, pp. 11-36, p. 14.
} 
Una de sus primeras "exploraciones" fue por las ciudades españolas, francesas y en el norte de Italia (Turín, Milán, Venecia y Trieste). Sus anotaciones de este viaje se encuentran en los Apuntes de un viaje. De España a Ginebra, redactados en 1873, junto a manuscritos que la escritora no quiso publicar ${ }^{20}$. Más tarde, su pasión por el viaje la llevó a entrar en contacto con las principales revistas españolas, y considerando la prensa como medio de difusión de las ideas y la más eficaz en su acción informativa, decidió publicar sus impresiones. En sus textos Emilia Pardo Bazán describe la realidad que está delante de sus ojos, y con ella hace buena literatura.

Durante la lectura del artículo de María Consuelo de Frutos Martínez ${ }^{21}$, mi atención se ha enfocado sobre el hecho de que la condesa haya decidido titular su libro Mi romería según el ejemplo de Dante en Vida Nova, aunque la referencia al escritor no se mencione de forma explícita:

no debería Ilamarse peregrinación, sino romería, este viaje. El uso, desde hace muchos años, corrompe el sentido de las palabras romería y romero, y así ocurre que se nombren romerías las fiestas celebradas en cualquier ermita o santuario a honra del patrón o de algún misterio religioso, y hay la romería de San Isidro y la de San Antonio, y en mi tierra la del Sacramento. Pero en rigor y con propiedad, romeros son los que van a Roma al jubileo o en cumplimento de voto; peregrinos los que in illo tempore se dirigían a Santiago de Compostela con las conchas y el bordón, y palmeros los que emprendían el camino de Jerusalén y regresaban con la palma en la mano. Permítaseme, pues, que de hoy más restituya a la excursión su verdadero nombre, Ilamándola romería siempre 22 .

La misma alusión a Dante la hace Manuel Curros Enríquez, en su obra de 1888 titulada $O$ divino sainete, de fuerte contenido satírico y anticlerical. De hecho, el texto está formado por una introducción y ocho cantos, en que los protagonistas (Curros y Añón) emprenden un viaje a Roma. Como la Divina Comedia de Dante, los dos personajes encuentran algunos vagones, en uno de éstos aparece también Doña Emilia, menospreciando a Rosalía de Castro junto con toda la literatura gallega.

Esta sátira currosiana depende sobre todo de que lo que Curros encuentra en el tren de los siete pecados es lo mismo que Emilia Pardo Bazán encuentra en el tren de romeros en Mi romería. La sátira consiste en que los personajes de Curros y Añón, en peregrinación para ver al papa, se encuentran en un tren Ileno de carlistas y religiosos

\footnotetext{
20 González Herrán, José Manuel, “Un inédito de Emilia Pardo Bazán: Apuntes de un viaje. De España a Ginebra”, en Literatura de viajes. El Viejo Mundo y el Nuevo, en S. García Castañeda (eds.), Madrid, Castalia, 1999, pp. 177-187.

21 Frutos Martínez de, Ma . Consuelo, “O divino sainete, de Curros Enríquez, e Mi romería, de Pardo Bazán”, en Boletín Galego de Literatura, n 43, 1 semestre 2010, Servizo de Publicacións Universidade de Santiago de Compostela, 2010, pp. 143-172.

22 Pardo Bazán, Emilia, Mi romería, en “Viajes por Europa”, ob. cit., p.41.
} 
integristas, de los que formaba parte Doña Emilia. De las palabras de María Consuelo de Frutos Martínez, se entiende que se trata de una segunda cara del viaje: la sátira de Curros, desde un punto de vista democrático, republicano, regionalista y anticlerical, es la respuesta a la visión católica y conservadora de Pardo Bazán. Y también el uso de la lengua gallega representa un punto importante, ya que la condesa considera el dialecto sólo para composiciones poéticas de carácter folclórico y popular.

Volviendo a la pasión de Doña Emilia por el viaje, es importante subrayar que ella sostiene el fin pedagógico del viaje también para los niños, y el efecto del contacto directo con las cosas: "instruir, estimular y abrir los horizontes de la vida a la infancia" ${ }^{23}$.

Hasta ahora solo se han encontrado estos datos, y es muy poco si se considera la amplia producción de la obra pardobazaniana. Lo mismo se puede decir con respecto a las traducciones al italiano, o a otros idiomas, de cuentos, novelas y ensayos, trabajo importante llevado a cabo por el profesor Danilo Manera, de la Universidad de Milán, Laura Silvestri, de la Universidad de Roma y Udine, y Tonina Paba, de la Universidad de Cagliari. Sin embargo, estas pequeñas consideraciones no bastan para colocar Italia en el primer lugar entre los países receptores de la obra de Emilia Pardo Bazán.

Esto sugiere la necesidad de un plan de colaboración para la difusión del tesoro literario que Doña Emilia nos ha dejado.

Me gustaría acabar este pequeño trabajo de resumen sobre el estado de la cuestión, citando unas palabras de la condesa, traducidas al italiano, de gran valor, íntimo y literario:

La mia qualità di signora mi ha fatto soffrire maggiormente per l'ipocrisia della critica e per le contraddittorie pretese del pubblico. lo sono, mi creda, una specie di amazzone, ma ho pure un carattere femminilissimo, che farci? Quando però mi si richiedono cose sciocche, io non so persuadermi che la mia condizione di signora abbia qualcosa da spartire con I'arte, e mettendomi a scrivere, dimentico che porto la gonna e mi sforzo di fare lavoro di artista e niente altro ${ }^{24}$.

23 Ibid., p. 18.

24 Paba, Tonina, Emilia Pardo Bazán e Italia, ob. cit., p. 534. Es la traducción al italiano de una carta que Luigi Capuana recibió de parte de la escritora coruñesa, y que está citada en su obra Gli ismi contemporanei de 1973: "Mi calidad de señora me hizo sufrir mayormente por la hipocresía de la crítica y de las contradictorias pretenciones del público. Yo soy, créame, una especie de amazona, pero también tengo un carácter muy femenino, ¿qué hacer? Pero cuando me piden cosas tontas, no sé convencerme de que mi condición de señora tenga algo que partir con el arte, y poniéndome a escribir, me olvido de que llevo una falda y me esfuerzo en hacer trabajo de artista, y nada más”. 


\section{BIBLIOGRAFÍA}

Frutos Martínez de, Ma Consuelo, (2010): “O divino sainete, de Curros Enríquez, e Mi romería, de Pardo Bazán", en Boletín Galego de Literatura, n 43, 1ºmestre 2010, Servizo de Publicacións Universidade de Santiago de Compostela, pp. 143 -172 .

Garosi, Linda, (2007): Poéticas de una crisis. La literatura italiana y española entre los siglos XIX y XX, Universidad de Córdoba, Facultad de Filosofía y Letras, Departamento de Traducción e Interpretación, Lenguas Romances, Estudios Semíticos y Documentación.

González Herrán, José Manuel, (1999): “Un inédito de Emilia Pardo Bazán: Apuntes de un viaje. De España a Ginebra", en Literatura de viajes. El Viejo Mundo y el Nuevo, en S. García Castañeda (eds.): Madrid, Ed. Castalia, 177-187.

Manera, Danilo, (2009): "Emilia Pardo Bazán en lengua italiana", José Manuel González Herrán, Cristina Patiño Eirín, Ermitas Penas Varela (eds.): Actas del Simposio "Emilia Pardo Bazán: estado de la cuestión", Casa-Museo Emilia Pardo Bazán, Real Academia Galega, Fundación Caixa Galicia, pp. 363-365.

Navarro Salazar, Ma Teresa, (1990): "La cuestión palpitante en Italia: Salvatore Farina y Emilia Pardo Bazán", en Epos (6), pp. 551-559.

Paba, Tonina, (2009): "Emilia Pardo Bazán e Italia", José Manuel González Herrán, Cristina Patiño Eirín, Ermitas Penas Varela (eds.): La literatura de Emilia Pardo Bazán, Casa-Museo Emilia Pardo Bazán, Real Academia Galega, Fundación Caixa Galicia, pp. 527-536.

Pardo Bazán, Emilia, (2009): La cuestión palpitante, La revolución y la novela en Rusia, La nueva cuestión palpitante, estudios de Laura Silvestri y Carlos Dorado, Editorial Bercimuel, Colmenar Viejo, Madrid.

Pardo Bazán, Emilia, (2006): Viajes por Europa, introducción y cronología de Tonina Paba (Universidad de Cagliari), Editorial Bercimuel, Colmenar Viejo, Madrid.

Sotelo Vázquez, Adolfo, (2004): "Emilia Pardo Bazán, Viajes por Europa", Intoducción de Tonina Paba, en La Tribuna. Cadernos de estudios da Casa-Museo Emilia Pardo Bazán, no 2, pp. 301-302.

Thion Soriano-Mollá, Dolores, (2005): "El epistolario de Doña Emilia Pardo Bazán, estado de la cuestión", José Manuel González Herrán, Cristina Patiño Eirín, Ermitas Penas Varela (eds.): Actas del Simposio "Emilia Pardo Bazán: estado de la cuestión", Casa-Museo Emilia Pardo Bazán, Real Academia Galega, Fundación Caixa Galicia, pp. 181-217. 


\section{WEBBIBLIOGRAFÍA}

www.realacademiagalega.org

www.cervantesvirtual.com

www.bibliotecamiralles.org 